Research Article

\title{
The Theory and Demonstration of the Solid-Fluid Transformation of Ice Water
}

\author{
Yong-Yan Wang $\mathbb{D}^{1},{ }^{1}$ Xi-Yan Fan $\mathbb{D}^{1,}{ }^{1,2}$ Nan Qin, ${ }^{1}$ Jian-Guang Li $\mathbb{D}^{1},{ }^{1}$ and Chuan-Qi Su${ }^{1}$ \\ ${ }^{1}$ Mechanics Center of Qingdao University of Science and Technology, Qingdao 266061, China \\ ${ }^{2}$ State Key Laboratory of Coal Resources and Safe Mining, China Mining University, Beijing 100083, China \\ Correspondence should be addressed to Yong-Yan Wang; wangyongyan168@163.com
}

Received 21 May 2020; Accepted 15 July 2020; Published 8 August 2020

Guest Editor: Zhi-Yuan Sun

Copyright (c) 2020 Yong-Yan Wang et al. This is an open access article distributed under the Creative Commons Attribution License, which permits unrestricted use, distribution, and reproduction in any medium, provided the original work is properly cited.

\begin{abstract}
In this paper, a mathematical model for describing the solid-fluid transformation of ice water is put forward based on the special geometry cases. The correctness of the obtained model is verified through comparison with numerical analysis and experiments. The good agreement indicates that the obtained model is available for the study of the solid-fluid transformation of ice water. The theory derived in this paper lays a foundation for the research of solid-fluid transformation phenomena of other materials and may have important applications in engineering areas such as rheology, creep, and instability of materials.
\end{abstract}

\section{Introduction}

The phenomena of solid-fluid transformation exist in the processes of ice melting, metal melting, rheology, and so on [1-7]. On September 11, 2001, the secondary collapse of the world trade center in the terrorist attack is a typical problem of creep under the action of thermal flow. The metals and concrete supports of the building crept under the action of thermal flow after a period of time, which led to the strength loss and collapse. Although the rheology and creep of materials have been hot topics in the engineering science, the mathematical aspect of the solid-fluid transformation has not been pointed out clearly. Starting from the Newton's law of cooling, the theory of the solid-fluid transformation of ice water will be derived in this paper. That can be a theoretical foundation for the solid-fluid transformation problem and may have important applications in the field of engineering science.

\section{The Mathematical Model for the Ice Water's Solid-Fluid Transformation}

Firstly, we take the ice ball as a special example to facilitate the deduction. Assume the radius of the ice ball as $r$, then the surface area of the ball as $4 \pi r^{2}$. Newton's law of cooling states that the rate of heat loss of a body is directly proportional to the difference between its own temperature and the temperature of its surroundings [8]. Therefore, the heat transferred through the surface of the ball within $\Delta t$ time can be expressed as follows:

$$
\Delta Q=4 \pi r^{2} \kappa\left(T-T_{0}\right) \Delta t
$$

where $\kappa$ is the heat transfer coefficient, $T_{0}$ is the temperature of ice ball, and $T$ is the temperature of its surroundings. It should be noted that we assume $T_{0}$ and $T$ are both constant and $T_{0}<0^{\circ} \mathrm{C}<T$. Within $\Delta t$ time, there are $\Delta V$ volumes of ice melting into water:

$$
\Delta V=4 \pi r^{2} \Delta r .
$$

Dividing the both sides of the above equation by $\Delta t$ and taking the limit, we have

$$
\lim _{\Delta t \rightarrow 0} \frac{\Delta V}{\Delta t}=4 \pi r^{2} \lim _{\Delta t \rightarrow 0} \frac{\Delta r}{\Delta t} .
$$


The above equation can be rewritten as

$$
\frac{d V}{d t}=4 \pi r^{2} \frac{d r}{d t}
$$

The density of ice is set to be $\rho$; then the quality of melted ice is $\Delta m=-\rho \Delta V$, where the negative sign signifies that the quality of ice ball decreases. The heat needed to convert $T_{0}$ ice to $0^{\circ} \mathrm{C}$ ice is

$$
\Delta Q_{1}=c \Delta m\left(0-T_{0}\right)=-\rho c \Delta V\left(0-T_{0}\right)=4 \pi r^{2} \rho c \Delta r T_{0},
$$

where $c$ denotes the specific heat of ice, namely, the heat needed to raise $1^{\circ} \mathrm{C}$ per unit mass of ice. The heat needed to melt $0^{\circ} \mathrm{C}$ ice to $0^{\circ} \mathrm{C}$ water can be calculated as follows:

$$
\Delta Q_{2}=\gamma \Delta m=-\rho \gamma \Delta V=-4 \pi r^{2} \rho \gamma \Delta r
$$

where $\gamma$ is the latent heat per unit mass of ice. Thus, the total amount of heat is

$$
\Delta Q=\Delta Q_{1}+\Delta Q_{2}=4 \pi r^{2} \rho\left(c T_{0}-\gamma\right) \Delta r
$$

By combining Equations (1) and (7), we have

$$
4 \pi r^{2} \rho\left(c T_{0}-\gamma\right) \Delta r=4 \pi r^{2} \kappa\left(T-T_{0}\right) \Delta t
$$

When $\Delta t \rightarrow 0$, the following differential equation could be derived:

$$
\frac{d r}{d t}=\frac{\kappa\left(T-T_{0}\right)}{\rho\left(c T_{0}-\gamma\right)}
$$

In order to integrate the above equation, we assume that the thermal parameters $c$ and $\gamma$ are both constant. Thus, we have

$$
r=\frac{\kappa\left(T-T_{0}\right) t}{\rho\left(c T_{0}-\gamma\right)}+C
$$

with $C$ as a constant of integration. When $t=0, r=r_{0}$, thus, we have $C=r_{0}$. When the ice ball is completely melted, i.e., $r=0$, the time of melting can be expressed as

$$
t=\frac{\rho\left(c T_{0}-\gamma\right)}{\kappa\left(T-T_{0}\right)} r_{0} .
$$

The denominator in the above equation is positive because $T>T_{0}$, while the numerator is negative because $T_{0}$ $<0^{\circ} \mathrm{C}$. Therefore, the time of melting $t$ is positive.

In fact, Equation (11) can also be obtained by the calculus relations. We assume that the thickness of ice ball melted within $d t$ time is $d r$; then the mass of ice melted is $d m=-\rho$ $d V=-4 \pi r_{2} \rho d r$. The heat of melting needed is

$$
d Q=\beta d m=-4 \pi r^{2} \rho \beta d r
$$

where $\beta$ is the heat of melting needed per unit mass of ice, with the unit J/kg. Based on Newton's law of cooling, the heat transferred through the surface of the ball within $d t$ time is

$$
d Q=4 \pi r^{2} \kappa\left(T-T_{0}\right) d t
$$

The following equation can be derived according to the conservation of energy:

$$
d Q=-4 \pi r^{2} \rho \beta d r=4 \pi r^{2} \kappa\left(T-T_{0}\right) d t .
$$

Then, the decreasing rate for the ice ball's radius with time $t$ is

$$
\frac{d r}{d t}=\frac{\kappa\left(T-T_{0}\right)}{\rho \beta} .
$$

The reduced surface area of ice ball within $d t$ time can be calculated as

$$
d S=4 \pi\left[(r+d r)^{2}-r^{2}\right]=4 \pi\left(2 r d r+d r^{2}\right)
$$

By ignoring the two-order infinitesimal, Equation (16) could be simplified as

$$
d S=8 \pi r d r .
$$

By dividing both sides of the above equation by $d t$, we can get the decreasing rate for the ice ball's surface area with time $t$ :

$$
\frac{d S}{d t}=8 \pi r \frac{d r}{d t}
$$

Substituting Equation (15) into Equation (18), the following equation can be obtained:

$$
\frac{d S}{d t}=-8 \pi r \frac{\kappa\left(T-T_{0}\right)}{\rho \beta}
$$

Although Equations (11), (15), and (19) are derived from the melting process of ice ball, they could apply to the general melting cases. For the cylinder, cone, and other shapes of ice, the corresponding equations can be derived in a similar way. In the following, we will take the ice cylinder as another example to derive the corresponding equations. Assume that the height of the ice cylinder is $h$ and the radius is $r$, then the surface area of the cylinder is $2 \pi r^{2}+2 \pi r h$. The heat transferred through the surface of the cylinder within $d t$ time can be expressed as

$$
d Q=\kappa\left(2 \pi r^{2}+2 \pi r h\right)\left(T-T_{0}\right) d t
$$

Within $d t$ time, there are $d V$ volumes of ice melting into water:

$$
d V=2 \pi r h d r+\pi r^{2} d h
$$


The heat needed to convert $T_{0}$ ice to $0^{\circ} \mathrm{C}$ ice is

$d Q_{1}=c d m\left(0-T_{0}\right)=-\rho c d V\left(0-T_{0}\right)=\rho c\left(2 \pi r h d r+\pi r^{2} d h\right) T_{0}$.

The heat needed to melt $0^{\circ} \mathrm{C}$ ice to $0^{\circ} \mathrm{C}$ water can be calculated as

$$
d Q_{2}=\gamma d m=-\rho \gamma\left(2 \pi r h d r+\pi r^{2} d h\right)
$$

Thus, the total amount of heat is

$$
d Q=d Q_{1}+d Q_{2}
$$
have

By combining Equations (20), (22), (23), and (24), we

$\kappa\left(2 \pi r^{2}+2 \pi r h\right)\left(T-T_{0}\right) d t=2 \pi r h \rho\left(c T_{0}-\gamma\right) d r+\pi r^{2} \rho\left(c T_{0}-\gamma\right) d h$.

The above mathematical relationships can be used as a unified theory of the solid-fluid transformation of ice water. That can be a theoretical foundation for the solidfluid transformation problem and may have important applications in the field of engineering science.

\section{Experimental Results on Ice Water's Solid- Fluid Transformation}

Ice melting is an unsteady heat transfer process, including not only heat conduction, convection, and radiation but also phase transformation. As for the influence factors regarding ice melting, the external influence factors such as environment temperature, humidity, pressure, convective heat transfer coefficient, and thermal radiation field play important roles, while the internal factors such as the temperature, shape, and size of ice also have nonnegligible effects [9]. The melting experiments were carried out by testing ice of different sizes and shapes in a RPH-80 thermotank, in which the environment temperature was set to be $25^{\circ} \mathrm{C}$. Specimens were prepared by pouring purified water into containers of different shapes and sizes, and then the containers were put in the freezer for 24 hours. The initial temperature of ice is set at $-18^{\circ} \mathrm{C}$. Table 1 shows the records of melting time of the ice with different shapes and sizes under the same experimental conditions.

\section{Comparison between the Mathematical Models with Experimental Results}

By processing the experimental data using Origin software, the linear fitting curves (as shown in Figure 1) and nonlinear (quadratic function) fitting curves (as shown in Figure 2) for the three types of specimens can be obtained, respectively. It can be seen that the experimental data agree well with the melting time of ice predicted through Equation (11). The melting time and diameter have similar relationship for three types of specimens, which further shows that Equation (11)
TABLE 1: The melting time for three kinds of shapes.

\begin{tabular}{lccc}
\hline Shape & $\begin{array}{c}\text { Diameter } \\
(\mathrm{mm})\end{array}$ & $\begin{array}{c}\text { Height } \\
(\mathrm{mm})\end{array}$ & $\begin{array}{c}\text { Time of } \\
\text { melting }(\mathrm{min})\end{array}$ \\
\hline \multirow{4}{*}{ Cylinder } & 25 & & 77 \\
& 35 & 100 & 96 \\
& 45 & 120 \\
& 55 & 155 \\
Circular truncated & 65 & 179 \\
cone & $25(\Phi 1), 40(\Phi 2)$ & 91 \\
& $45(\Phi 1), 60(\Phi 2)$ & 100 & 116 \\
& $55(\Phi 1), 70(\Phi 2)$ & 144 \\
& $65(\Phi 1), 80(\Phi 2)$ & 175 \\
& 25 & 215 \\
\hline \multirow{4}{*}{ Ball } & 35 & 42 \\
& 45 & 63 \\
& 55 & 101 \\
& 65 & 125 \\
\hline
\end{tabular}

also works for the cases of the cylinder and circular truncated cone. The differences on slopes and curvatures of fitting curves show that the heat transfer coefficient is a comprehensive parameter, which changes with the shape and size of specimen. Two kinds of fitting are analyzed by the way of comparative error analysis, as shown in Figure 3, from which it can be seen that the nonlinear fitting error is smaller than the linear fitting case for the relationship between the melting time and the diameter of the ice ball cylinder and circular truncated cone. The heat transfer coefficients for the ice ball, cylinder, and circular truncated cone are unrelated to their diameters.

\section{Numerical Analysis on the Solid-Fluid Transformation of Ice Water}

In the following, ANSYS and fluent analyses will be employed to study the process of ice melting numerically $[10,11]$. The mesh size used in the software is $3 \mathrm{~mm}$, and the number of nodes for the ice ball is 1766 , the ice cylinder is 5888 , and the ice circular truncated cone is 8014 .

5.1. ANSYS Numerical Simulation Analysis. Ice melting is a process related to the phase transformation. The latent heat must be taken into consideration when the phase transformation is involved, because the latent heat is defined from the concept of enthalpy, which will be taken as an attribute definition of material. The curve that enthalpy changes with temperature can be obtained. According to the definition, solid temperature $\left(T_{s}\right)$ and fluid temperature $\left(T_{l}\right)$ will divide the enthalpy curve into three areas, i.e., the solid area $\left(T<T_{s}\right)$, phase-transition area $\left(T_{s}<T<T_{l}\right)$, and fluid area $\left(T_{l}<T\right)$. The phases can be distinguished by the temperatures in different time. The temperature of ice for phase transformation is $0^{\circ} \mathrm{C}$ in the atmospheric circumstances. 


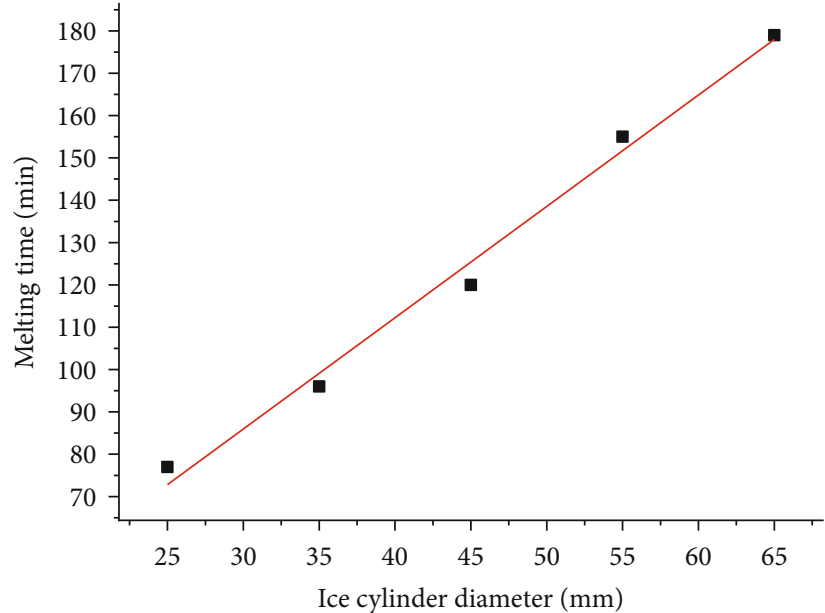

- Different melting time

— Fitting straight-line

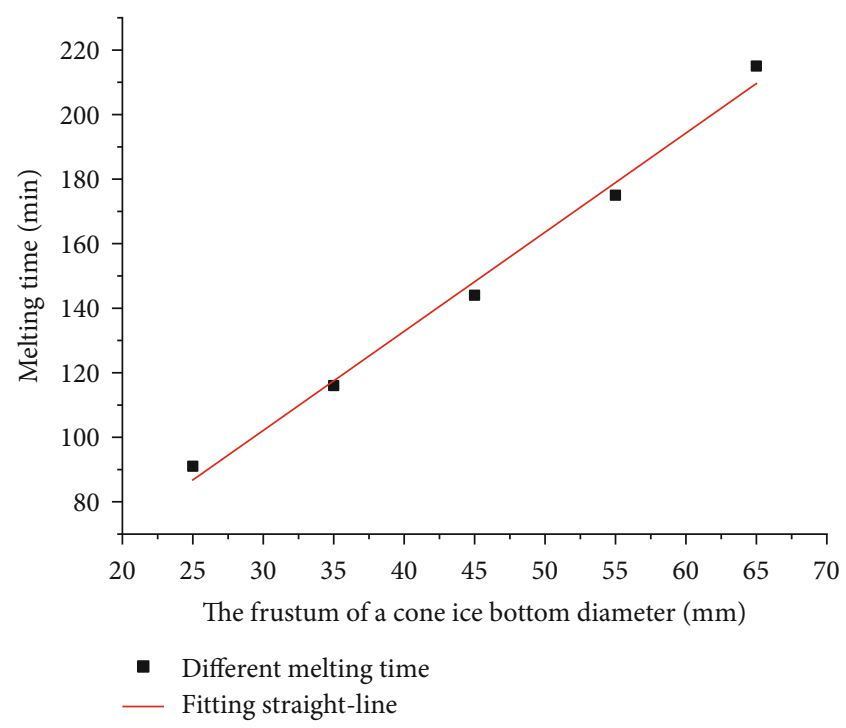

(b)

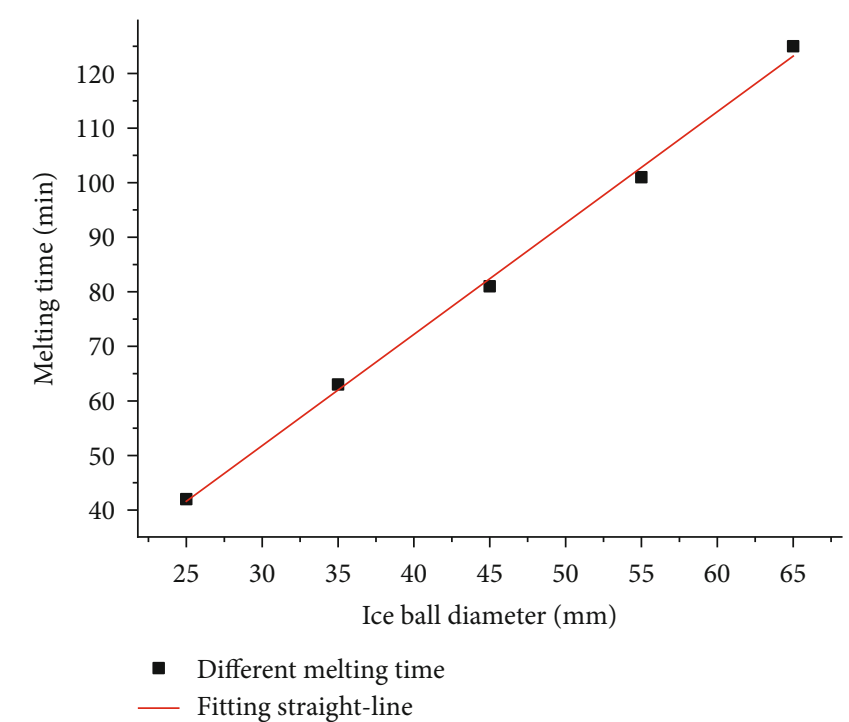

(c)

Figure 1: The linear fit between the melting time and diameter: (a) ice cylinder, (b) ice circular truncated cone, and (c) ice ball.

When the temperature is higher than $0^{\circ} \mathrm{C}$, the material will melt from ice to water [10].

The melting process of ice in the thermotank is mainly influenced by the heat convection and radiation. Typical parameters for the thermotank wall are shown in Table 2. The temperature is set to be $25^{\circ} \mathrm{C}$ in the thermotank, and the initial temperature of ice is $-18^{\circ} \mathrm{C}$. Considering the heating principle and the heat transfer process of ice in thermotank, we set the mixed boundary condition on the surface of ice that contacts with air and selects the convective heat transfer coefficient as $20 \mathrm{~W} /\left(\mathrm{m}^{2} \cdot \mathrm{K}\right)[10]$.

From the numerical simulation results worked by ANSYS, we find that the temperature of ice ball reduces from the external to the inner on the melting nephogram at $60 \mathrm{~min}$, which agrees perfectly with the experimental results in the thermotank. The temperature of the ice cylinder and circular truncated cone also reduces from the external to the inner part on the melting nephogram at $60 \mathrm{~min}$, and the temperature at the edges and angles remains the highest, indicating that the melting process starts from the edges and angles to the inner gradually. The results of the numerical simulation also agree well with that of the corresponding experiments in the thermotank. Table 3 shows the melting time in the numerical simulation and the thermotank experiment, respectively. It can be seen from Table 3 that the agreement degree between numerical and experimental results on the melting time for ice ball, cylinder, and circular truncated cone reaches $92.6 \%, 92.5 \%$, and $95.3 \%$, respectively. The melting time for the numerical simulation is shorter than that for the experiment, which can be attributed to the loss of heat during the experiment.

5.2. Fluent Numerical Simulation Analysis. In order to further verify the correctness of the theoretical model and make 


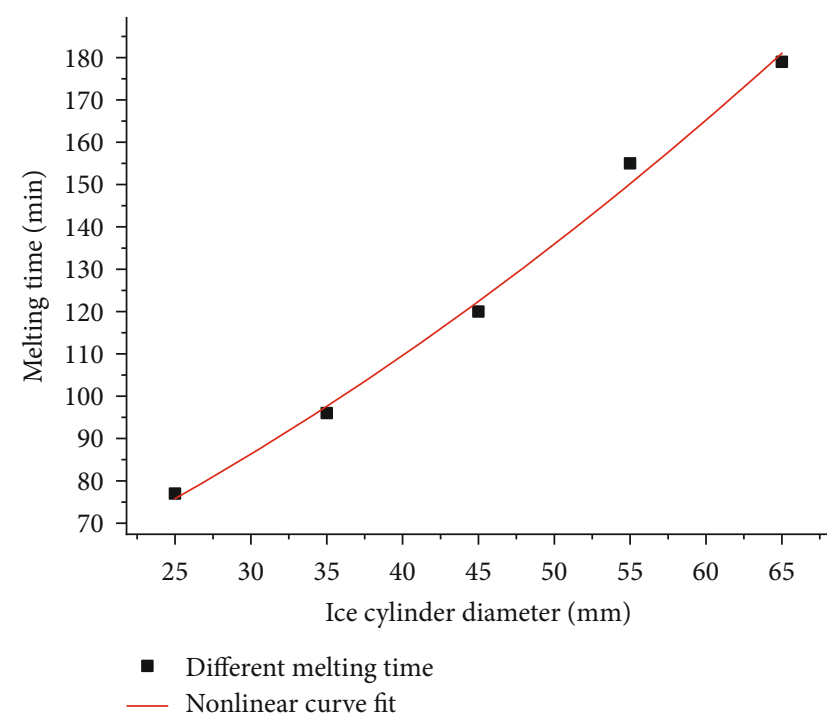

(a)

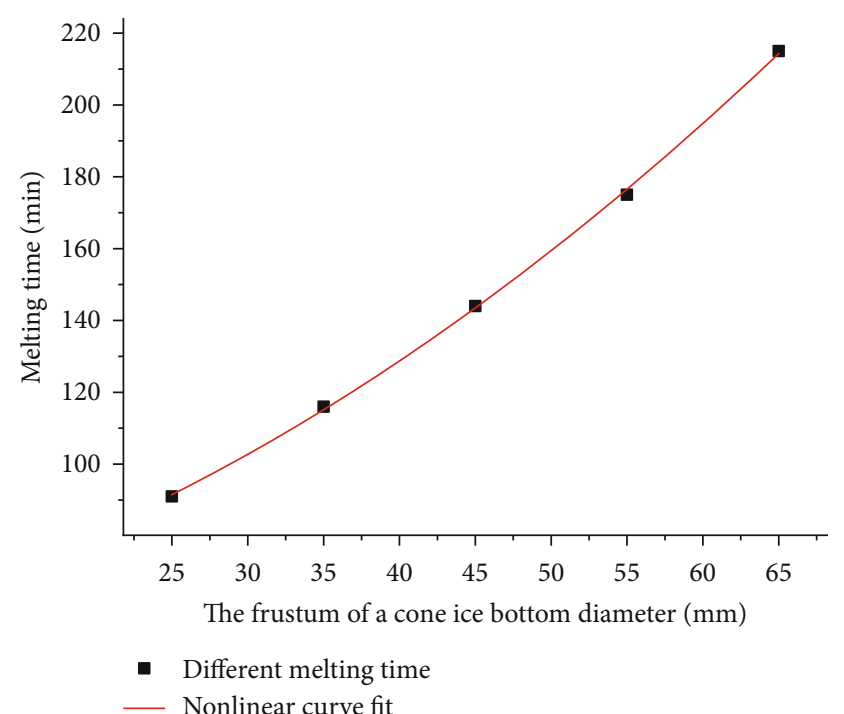

(b)

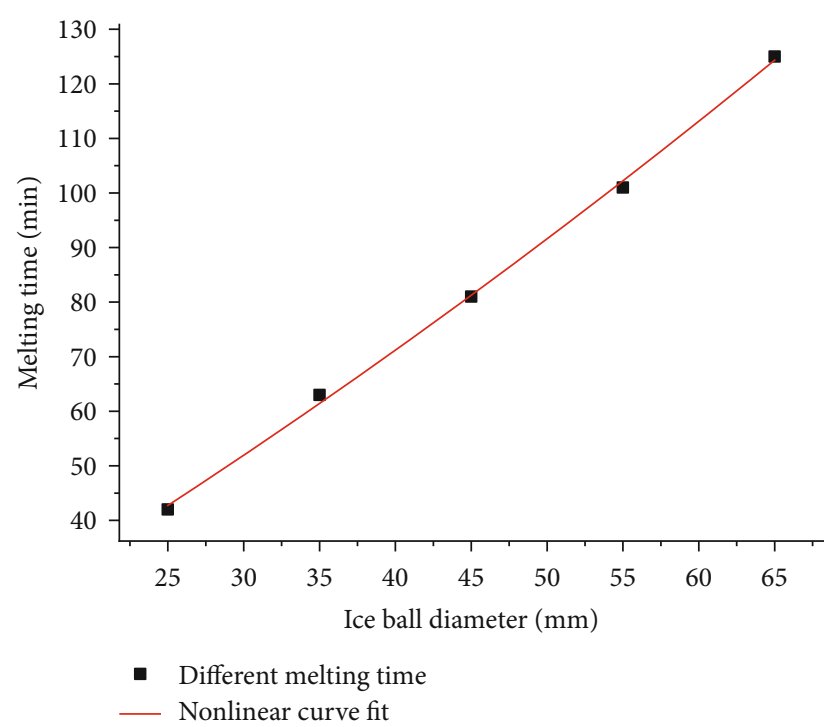

(c)

Figure 2: The nonlinear fit between the melting time and diameter: (a) ice cylinder, (b) ice circular truncated cone, and (c) ice ball.

a comparison with the results of ANSYS simulation, fluent analysis is employed to simulate the melting process of ice. The air medium in the box is set to be ideal gas, and environmental pressure is set as a standard atmospheric pressure. The latent heat of ice medium is $333146 \mathrm{~J} / \mathrm{kg}$, and the melting point of ice and freezing point of water are the same $(273.15 \mathrm{~K})$ [11].

Fluent analysis uses the heat enthalpy technology to handle with the melting and solidification processes. The melting process is characterized by the changing of the liquid fraction without tracing on the interface [11]. The simulation results are shown in Figure 4 and Table 4, from which it can be seen that the melting processes start from the corners, then carry on from the outside to the inside. The simulation results by fluent analysis also agree well with experiments and are consistent with the results worked out by ANSYS.
It can be seen from Table 4 that the melting time for the ice ball, cylinder, and circular truncated cone by the Fluent simulation is less than that by the experiments and the rates of agreement reach $91.4 \%, 95.8 \%$, and $97.2 \%$ for the ice ball, cylinder, and circular truncated cone, respectively. The reason for this phenomenon is that in the numerical simulation, the boundary conditions and material properties are in the ideal conditions and there is no heat loss.

\section{Conclusion}

In this paper, the theory of the solid-fluid transformation of ice water with special shapes such as the ball, cylinder, and circular truncated cone has been derived and the mathematical relationships of ice water's solid-fluid transformation have been presented. The unified theory and formulas of 


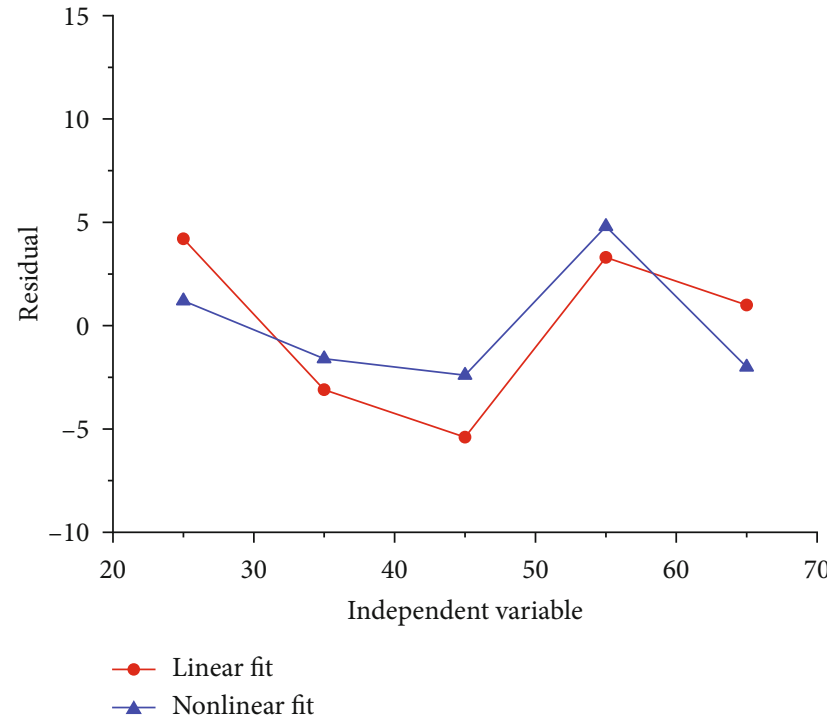

(a)

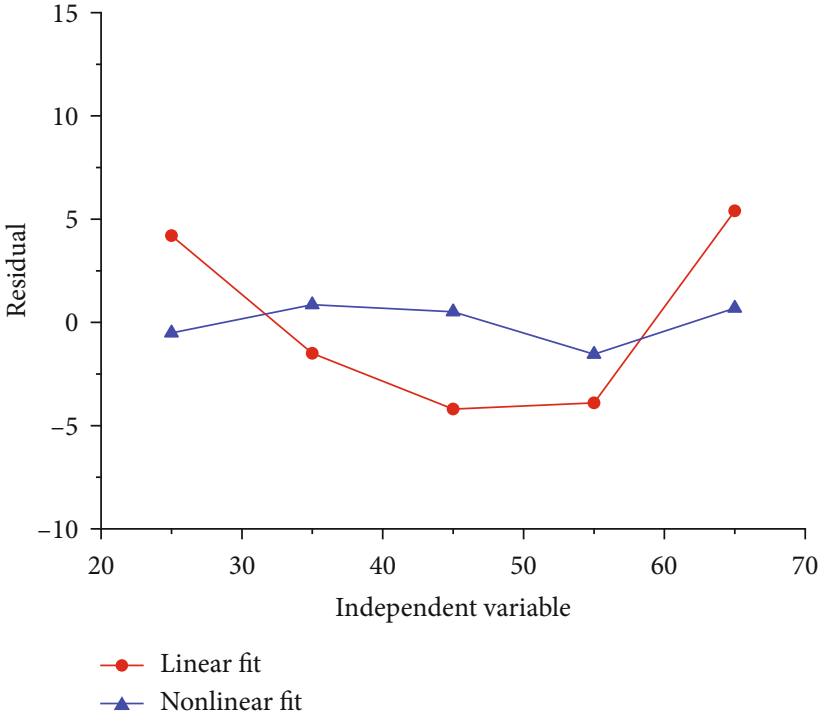

(b)

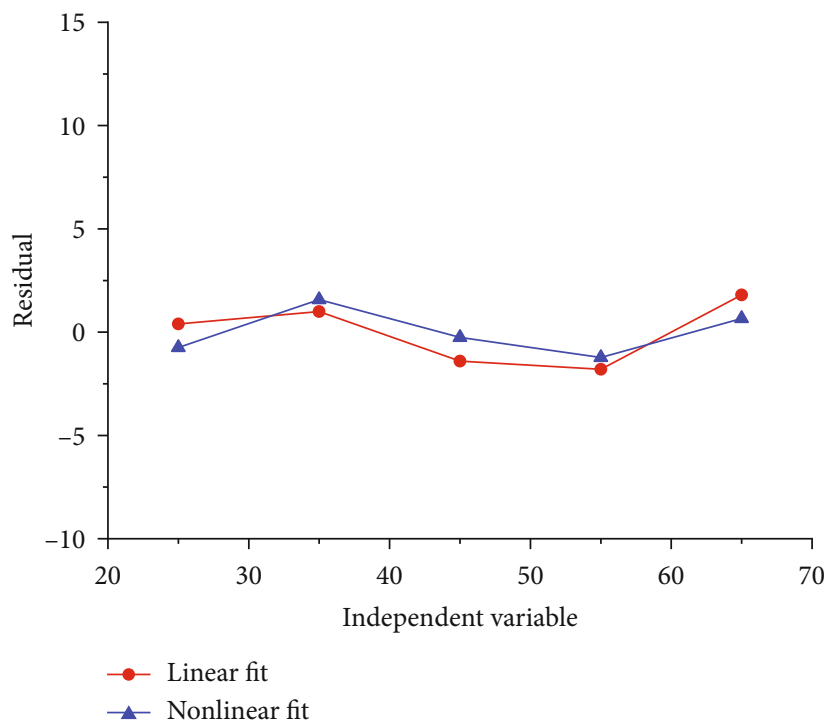

(c)

FIgURE 3: The comparative error analysis for the two kinds of fitting: (a) ice cylinder, (b) ice circular truncated cone, and (c) ice ball.

TABLE 2: Typical parameters for the thermotank wall.

\begin{tabular}{lcccc}
\hline $\begin{array}{l}\text { Coefficient of thermal } \\
\text { conductivity }[\mathrm{W} /(\mathrm{m} \cdot \mathrm{K})]\end{array}$ & $\begin{array}{c}\text { Density } \\
\left(\mathrm{kg} / \mathrm{m}^{3}\right)\end{array}$ & $\begin{array}{c}\text { Modulus of } \\
\text { elasticity }(\mathrm{Pa})\end{array}$ & $\begin{array}{c}\text { Poisson's } \\
\text { ratio }(1)\end{array}$ & $\begin{array}{c}\text { Specific heat } \\
\text { capacity }[\mathrm{J} /(\mathrm{kg} \cdot \mathrm{K})]\end{array}$ \\
\hline 60.64 & 7850 & $2 \mathrm{e} 11$ & 0.3 & 96 \\
\hline
\end{tabular}

TABLE 3: The comparison of melting time between ANSYS simulation and experimental results.

\begin{tabular}{lccccc}
\hline Shape & Size $(\mathrm{mm})$ & & ANSYS $(\min )$ & Experiment $(\mathrm{min})$ & Difference $(\%)$ \\
\hline Ball & Diameter & 45 & 75 & 120 & 7.4 \\
\hline Cylinder & Diameter & 45 & 111 & & 7.5 \\
& Height & 100 & 45 & & 144 \\
\hline Circular & Top & 60 & 137 & & 4.7 \\
Truncated & Root & 100 & & & \\
Cone & Height & & & & \\
\hline
\end{tabular}




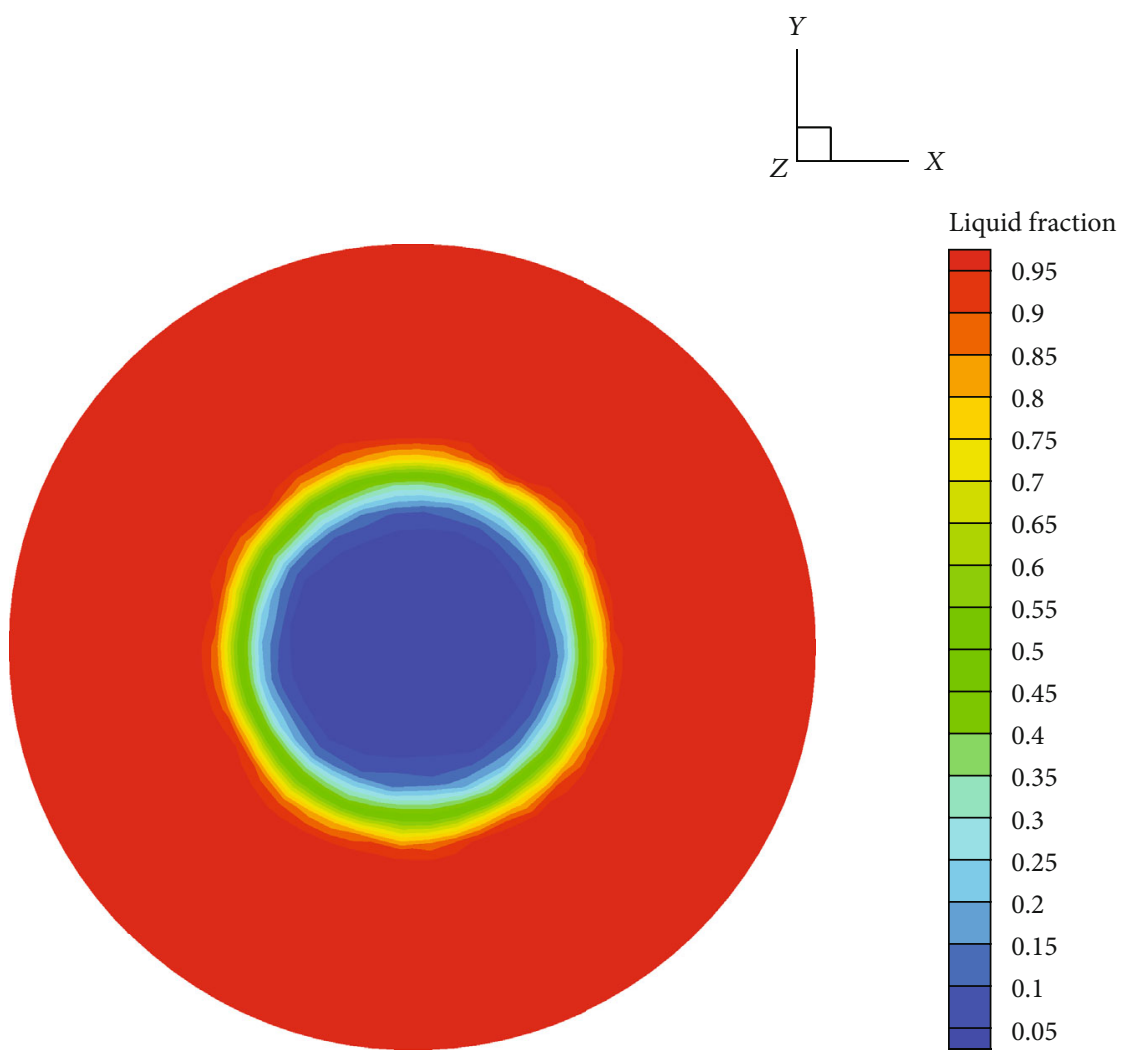

FIgURE 4: The simulation result for the ice ball by fluent analysis at $60 \mathrm{~min}$.

TABLE 4: The comparison of melting time between fluent simulation and experimental results.

\begin{tabular}{lccccc}
\hline Shape & Size $(\mathrm{mm})$ & & Fluent $(\mathrm{min})$ & Experiment $(\mathrm{min})$ & 81 \\
\hline Ball & Diameter & 45 & 74 & 120 & Difference $(\%)$ \\
\hline Cylinder & Diameter & 45 & 115 & & \\
& Height & 100 & 45 & & 144 \\
\hline Circular & Top & 60 & 140 & & 2.8 \\
Truncated & Root & 100 & & & \\
Cone & Height & 100 & & \\
\hline
\end{tabular}

ice water's solid-fluid transformation are validated through simulation analysis and corresponding experiments. It is expected that the theory derived in this paper could lay a foundation for the research of solid-fluid transformation phenomena of other materials and may have important applications in engineering areas.

\section{Data Availability}

The data used to support the findings of this study are available from the corresponding author upon request.

\section{Conflicts of Interest}

The authors declare that they have no conflicts of interest.

\section{Acknowledgments}

We express our sincere thanks to the editors, reviewers, and all the members of our discussion group for their valuable comments. This work has been supported by the National Natural Science Foundation of China under Grant No. 51674149, Open Research Fund of the State Key Laboratory of Coal Resources and Safe Mining under Grant No. SKLCRSM20KF006 and Natural Science Foundation of Shandong Province under Grant No. ZR2019MEE082.

\section{References}

[1] W. K. Heidug, "Intergranular solid-fluid phase transformations under stress: the effect of surface forces," Journal of Geophysical Research: Solid Earth, vol. 100, no. B4, pp. 5931-5940, 1995. 
[2] J. Ghoussoub and Y. M. Leroy, "Solid-fluid phase transformation within grain boundaries during compaction by pressure solution," Journal of the Mechanics and Physics of Solids, vol. 49, no. 10, pp. 2385-2430, 2001.

[3] J. I. D. Alexander and W. C. Johnson, "Thermomechanical equilibrium in solid-fluid systems with curved interfaces," Journal of Applied Physics, vol. 58, no. 2, pp. 816-824, 1985.

[4] A. Putnis and C. V. Putnis, "The mechanism of reequilibration of solids in the presence of a fluid phase," Journal of Solid State Chemistry, vol. 180, no. 5, pp. 1783-1786, 2007.

[5] A. S. Dogonchi, M. Hatami, and G. Domairry, "Motion analysis of a spherical solid particle in plane Couette Newtonian fluid flow," Powder Technology, vol. 274, pp. 186-192, 2015.

[6] J. Yu, X. Chen, H. Li, J. W. Zhou, and Y. Y. Cai, "Effect of freeze-thaw cycles on mechanical properties and permeability of red sandstone under triaxial compression," Journal of Mountain Science, vol. 12, no. 1, pp. 218-231, 2015.

[7] G. Wang, X. Qin, J. Shen, Z. Zhang, D. Han, and C. Jiang, "Quantitative analysis of microscopic structure and gas seepage characteristics of low-rank coal based on CT threedimensional reconstruction of CT images and fractal theory," Fuel, vol. 256, p. 115900, 2019.

[8] S. M. Yang and W. Q. Tao, Heat Transfer, Higher Education Press, Beijing, 2006.

[9] M. E. Grainger and H. Lister, "Wind speed, stability and eddy viscosity over melting ice surfaces," Journal of Glaciology, vol. 6, no. 43, pp. 101-127, 1966.

[10] W. T. Xin, Z. Z. Li, and R. X. Hu, ANSYS13.0 Thermodynamic Finite Element Analysis, Machinery Industry Press, Beijing, 2011.

[11] J. P. Tang, FLUENT 14.0 Super Learning Manual, The Posts and Telecommunications Press, Beijing, 2013. 